Cite this: RSC Advances, 2013, 3, 2662

Received 28th August 2012, Accepted 7th December 2012

DOI: $10.1039 / \mathrm{c} 2 \mathrm{ra} 21963 \mathrm{~h}$

www.rsc.org/advances

\title{
The critical role of surfactants towards CdS nanoparticles: synthesis, stability, optical and PL emission properties
}

\author{
Sanjay Kumar, ${ }^{\text {ab }}$ Michael Gradzielskic and S. K. Mehta*a
}

\begin{abstract}
Cadmium sulfide (CdS) nanoparticles (NPs), prepared by a convenient chemical precipitation method, have been characterized using techniques such as TEM, XRD, zeta potential, absorption and photoluminescence (PL) emission spectroscopy to establish the structure directing role of different cationic and anionic surfactants and their impact on the nanoparticles stabilization. In the synthesis of the CdS NPs, cadmium acetate and sodium sulfide, employed as starting reagents, were dissolved in aqueous solutions of different surfactants to study the effect of their structures on the nucleation, growth, optical and PL emission properties of the NPs. By varying the surfactant species, the CdS NPs have significantly different optical and PL emission properties despite being produced under similar reaction conditions. Depending on the surfactant structure, the growing CdS NPs were stabilized by the surfactants to different extents. For example, in the surfactant with the longest chain length (e.g. cetyltrimethylammonium bromide; $(T A B)$, the CdS NPs were most stable, whereas using a surfactant with a smaller chain length i.e. DTAB, the NPs were unstable for even $1 \mathrm{~h}$. On the other hand, anionic surfactants of even smaller chain lengths were able to stabilize the CdS NPs for quite long times. The generalized study of growth of spherical CdS NPS involves monitoring the kinetics during the progress of the reaction. Additionally, an interesting prominent effect of surfactant structure on the PL emission properties of the NPs has been established under identical reaction conditions.
\end{abstract}

\section{Introduction}

In the past decade, semiconductor nanoparticles (NPs) have gained tremendous popularity because of their numerous technological applications ranging from biological labels ${ }^{1,2}$ to optoelectronic transistor components. ${ }^{3}$ Many important stu$\operatorname{dies}^{4-6}$ on CdS NPs have revealed a Bohr radius of $2.4 \mathrm{~nm}^{7}$ and direct band gap of $2.42 \mathrm{eV}{ }^{8}$ The leading application areas of these nanomaterials include photovoltics, light emitting diodes and other optical devices. ${ }^{9,10}$ Since most of the properties of semiconductor NPs strongly depend on the size, size distributions and crystal structures, ${ }^{11}$ the majority of the research has been limited to shape and size manipulations of CdS NPs. For example, CdS NPs of various shapes and morphologies, such as nanowires, ${ }^{12}$ triangular and hexagonal CdS NCs, ${ }^{13-15}$ nanoflowers and nanotrees, ${ }^{16}$ belts $^{17,18}$ and

\footnotetext{
${ }^{a}$ Department of Chemistry and Centre for Advanced Studies in Chemistry, Panjab University, Chandigarh-160014, India.E-mail: skmehta@pu.ac.in; Fax: +91 172 2545074; Tel: +91 1722534423

${ }^{b}$ Department of Chemistry, Government College, Chowari (Chamba), H.P. -176302, India

${ }^{c}$ TU Berlin, Institut für Chemie, Stranski-Laboratorium für Physikalische Chemie und Theoretische Chemie, Sekr. TC 7, Strasse des 17. Juni 124, D-10623 Berlin, Germany † Electronic supplementary information (ESI) available. See DOI: 10.1039/ c2ra21963h
}

tetrapods, ${ }^{19}$ have been synthesized by laser ablation, thermal evaporation, ${ }^{20,21}$ solution chemistry routes $^{22}$ and templating $^{23,24}$ etc. CdS nanorods have been prepared by a solvothermal process ${ }^{25-28}$ using various capping agents or a passivator to control and induce the growth of NCs. Despite these numerous reports, many aspects related to the formation of CdS nanostructures in aqueous media, stabilized through various organic surfactants, remain to be explored. Among the few studies, Mehta et al. ${ }^{29}$ have carried out various investigations related to the synthesis and growth of CdS NPs stabilized with different cationic surfactants in aqueous media. Pandey et $a .^{30}$ have studied the effect of the reaction time and surfactants (cetyltrimethyl ammonium bromide (CTAB) and sodium dodecyl sulfate (SDS)) on the morphology and size of the CdS NPs to show dramatic shape and size variations by varying the surfactant and reaction time. Chakraborty et al. ${ }^{31}$ have utilized a very simple approach to prepare nanodispersions (colloids) of $\mathrm{CdS}$ and $\mathrm{HgS}$ as well as their core-shell products and composites (co-colloids) in micellar solutions of the cationic surfactant CTAB. A relatively simple chemical method to synthesize CdS nanotubes by employing the surfactants AOT and Triton-X have been developed by Rao et $a l^{32}$ To improve the application potential of these synthetic routes, further advancement is an important issue which 
needs to be considered through elaborating the tailoring of the nanocrystal morphology, elucidating the growth mechanism and optimizating the physical parameters for the generation of well defined nanostructures.

In addition to interesting optical properties, CdS NPs with a diameter of less than $10 \mathrm{~nm}$ have become a recent topic of intense investigations towards their luminescent properties mainly due to their inherent broad PL spectrum. ${ }^{33}$ The formation of CdS nanostructures in aqueous media is a chemical process initiated by the reactions of molecules or ions that serve as precursors to nuclei. Nuclei undergo growth or further reactions to form nanostructures. In these cases of nanostructure evolution, the luminescent efficiency of the NPs are strongly dependent on the surface state and could be significantly improved by altering the surface passivation. ${ }^{34,35}$ Therefore, probing of surface state of CdS NPs is very important due to either a theoretical interest or the instructive significance in many applications.

In this work, we report various investigations related to the stabilization of CdS NPs synthesized in aqueous media with different cationic and anionic surfactants as stabilizing agents. The objective of the study is to provide a sufficient database of various parameters related to the synthesis and stabilization of surfactant stabilized CdS NP through comparative investigations on the nucleation and growth in the presence of different surfactants under identical conditions. Additionally, these investigations could be useful in understanding the origin of the optical and PL emission properties of the CdS NPs with respect to the studied surfactants. Fig. S1 (ESI $\dagger$ ) depicts the molecular structures of the various surfactants used in this study.

\section{Results and discussion}

\subsection{TEM and DLS (dynamic light scattering) measurements}

The sizes and morphologies of the CdS NPs $(0.7 \mathrm{mM})$ stabilized in different surfactants $(3 \mathrm{mM})$ have been assessed using TEM. Fig. 1 depicts a collection of TEM images. All the NPs appear to be nearly spherical except for those synthesized in DTAB. Virtually monodispersed and well-separated CdS NPs are evident in all the cationic surfactants as well as in SL. However, the NPs exist as a flower-like pattern in SDS and SDBS, where 8-10 NPs seem to be closely associated. The aqueous dispersions of the CdS NPs in these two surfactants were equally homogeneous, as for the other surfactants, and therefore these patterns of the NPs are considered to be generated during the sample drying process on copper grids due to the different natures of the surfactants.

A little distortion from the spherical shape has also been noticed for the CdS NPs synthesized in TTAB compared to those synthesized in other cationic surfactants. This may be due to the comparatively weak stabilizing tendency of TTAB. Furthermore, DTAB could not sufficiently stabilize the CdS NPs, leading to the formation of irregular shaped, bigger sized particles (Fig. 1). Still, the DTAB stabilized NPs are well dispersed and are in the nm size range as well. The particle sizes presented in Table 1 have been derived from the TEM images by averaging the sizes of 30-40 NPs. The sizes and shapes of the CdS NPs have been found to be strongly surfactant dependent.

The size distributions of the aqueous CdS NPs (already analyzed through TEM) have been obtained from DLS measurements. The CONTIN algorithm was applied to intensity autocorrelation data to obtain the intensity weighed size distributions of the samples and the profiles are shown in Fig. S2 (ESI $\dagger$ ). Evidently, bimodal distributions with one major and a minor peak have been observed in all the cases. The

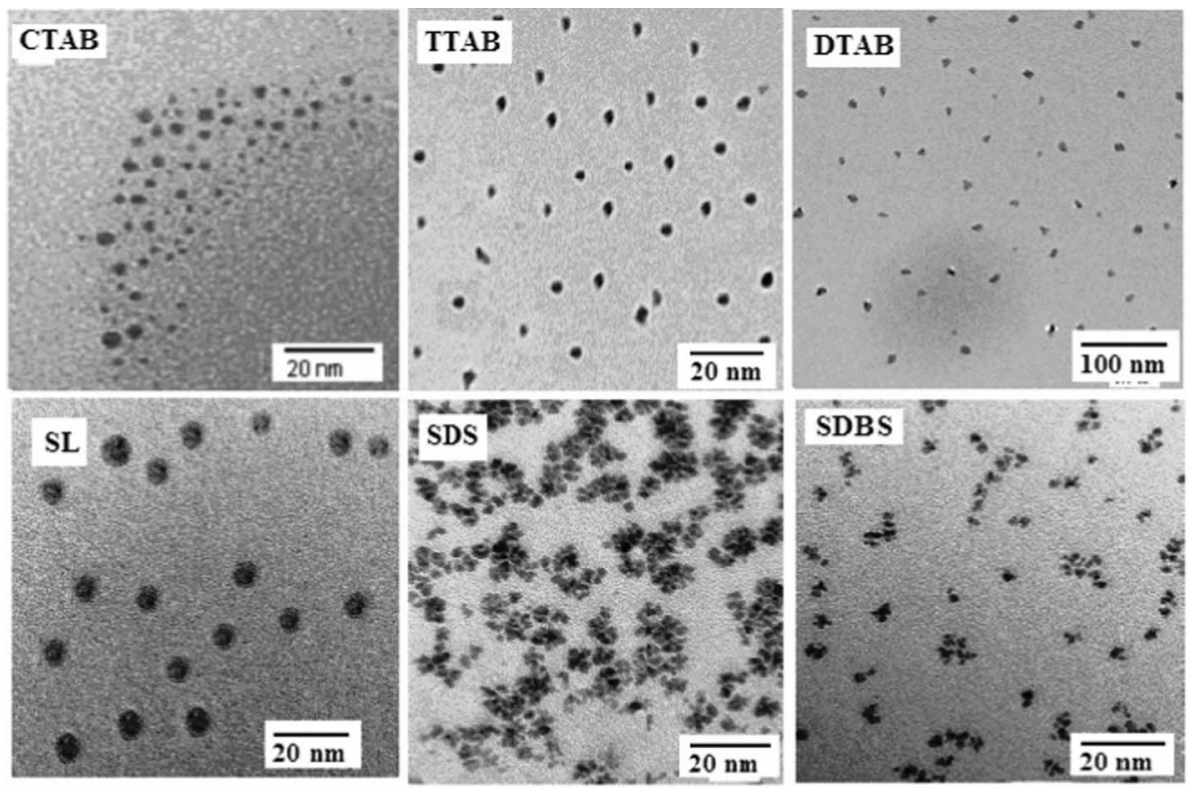

Fig. 1 TEM images of the CdS NPs $(0.7 \mathrm{mM})$ stabilized in aqueous solutions of different surfactants (3 mM). 
Table 1 The sizes and PDI of the surfactant stabilized CdS NPs deduced from the TEM and DLS measurements

\begin{tabular}{llll} 
[Surfactant] $(3 \mathrm{mM})$ & $d_{\text {TEM }}(\mathrm{nm})( \pm 0.5)( \pm$ standard deviation $)$ & Hydrodynamic radii $(\mathrm{nm})$ obtained from DLS $( \pm$ standard deviation $)$ & PDI $(\mathrm{DLS})$ \\
\hline CTAB & $2.70 \pm 1.04$ & $8.60 \pm 3.80$ & 0.362 \\
TTAB & $3.10 \pm 0.93$ & $12.4 \pm 4.60$ & 0.410 \\
DTAB & $32.5 \pm 6.94$ & $35.1 \pm 11.40$ & 0.570 \\
SL & $3.15 \pm 0.45$ & $9.40 \pm 3.10$ & 0.384 \\
SDS & $3.00 \pm 1.12$ & $10.4 \pm 3.20$ & 0.416 \\
SDBS & $2.60 \pm 1.44$ & $7.90 \pm 2.90$ & 0.311
\end{tabular}

intensity and dispersity of each peak significantly depends upon the surfactant type. Fig. S2 (ESI†) clearly indicates the presence of a few bigger cluster formations, though most of the NPs are in a 4 to $22 \mathrm{~nm}$ range in all the surfactants except TTAB and DTAB. The NPs in TTAB and DTAB have size ranges of 8-40 $\mathrm{nm}$ and $16-85 \mathrm{~nm}$, respectively. The size distributions of the CdS NPs have been observed to be narrowest in SDBS and broadest in DTAB. The average particle sizes, corresponding to the maxima of the most intense peak in the DLS size distribution profiles, have been given in Table 1 .

This technique measures the hydrodynamic radius of the nanodispersions which includes the size of the adsorbed surfactant layer/micelles and hydration shell of the head group as well. However, TEM measures the size of the CdS cores only, without taking into account their surroundings. The radius of the DTAB and CTAB aqueous micelle with a $\mathrm{C}_{12}$ and $\mathrm{C}_{16}$ carbon tail length has been reported to be $1.8 \mathrm{~nm}$ and $2.3 \mathrm{~nm}$ respectively, though it may vary in the presence of inorganic salts. ${ }^{36}$ Therefore, the small disagreement of at least $4-7 \mathrm{~nm}$ in the sizes of the CdS NPs obtained from the DLS and other techniques is justifiable.

\subsection{X-ray powder diffraction (XRD)}

The crystal structure of the powdered CdS NPs obtained after solvent evaporation from different surfactants has been established with the help of XRD analysis and the typical diffractograms are shown in Fig. 2. The XRD peaks at the $2 \theta$ angles of $25.7^{\circ}, 43.8^{\circ}$ and $52.0^{\circ}$ have been indexed to the $<100\rangle,<220\rangle$ and $<311>$ planes, respectively. On comparing these with the standard JCPDS database values, all the peaks can be indexed to pure cubic CdS. Interestingly, all the XRD patterns of CdS have been found to be almost the same, irrespective of the surfactants used in the synthesis. This obviously indicates that all the surfactants give NPs with the same average size in powder form and that the structure dependent tendencies of the surfactants towards stabilization of the NPs are significant only in aqueous solutions.

On the basis of similar XRD studies, Chae et al. ${ }^{37}$ have reported the production of spherical and rod shaped CdS NPs and found that the diffraction patterns have been found to be dependent on the NPs shape. On comparing the diffraction patterns with those of various shaped NPs, the diffraction patterns can be taken as indicative of the formation of spherical CdS NPs in all the surfactants. From the broadening of the XRD peaks, the sizes of the nanocrystallites were

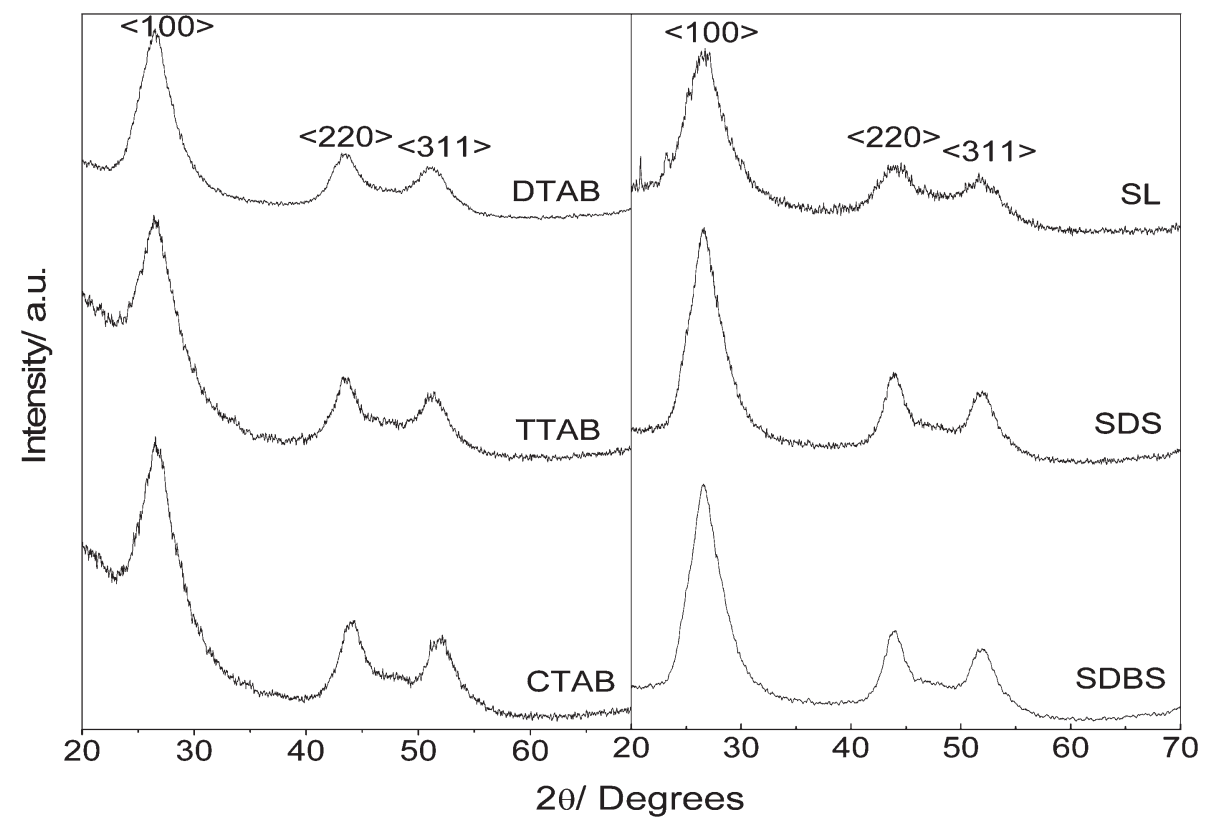

Fig. 2 XRD patterns of the powdered ZnS NPs recovered from aqueous solutions of different surfactants. 
calculated using the Debye-Scherrer formula ${ }^{38}$ and the average crystallite size has been found to be $2.1 \pm 0.1 \mathrm{~nm}$. The sizes of the NPs, as obtained from the powder XRD patterns, underestimate the size obtained by TEM spectroscopy. Qualitatively, this disagreement of sizes calculated using different techniques is justified as the XRD line broadening does not take into account the other contributions e.g. lattice defects, dislocations, faulting and lattice strain. ${ }^{39}$ It is remarkable in this study that the primary nanocrystal in the powder form has the same size and structure irrespective of the surfactant, which in an aqueous solution exists as an ensemble of many NCs which form NPs of varying sizes. Since the XRD analysis reveals that the CdS NPs have almost identical natures in the powder form, the morphology of some selected samples has been investigated through SEM. Fig. S3 (ESI†) presents the SEM images of the powdered CdS NPs separated from some cationic and anionic surfactants. The images clearly depict spherical particles with some aggregates in CTAB, whereas highly associated irregular-shaped particles are evident in the sample separated from DTAB. Spherical and associated particles have also been noticed in SDBS with presence of some surfactant layers in between the particles. The magnified view of such a single particle has been presented in Fig. S3(d). $\dagger$ One can clearly interpret that the large sized single particle in te SEM image is actually an ensemble of many small spherical particles which have associated with one another during the solvent evaporation. This also justifies the large sizes of the CdS NPs in the powder form measured from the SEM images.

\subsection{UV-vis spectroscopic analyses: optical band gap and size determination}

The UV-vis absorption spectra of the as-prepared dispersions of the CdS NPs have been recorded after one hour and the spectra are shown in Fig. 3(a). Each spectrum shows an absorption onset in the range of $470-490 \mathrm{~nm}$. The exact positions of the spectral absorption edges of the CdS NPs in different surfactants have been given in Table 2 .

Compared to the UV-absorption of the bulk (540 nm) material, the apparent blue shift in the absorption edges of the CdS NPs indicates that the position dependent quantum size effect has been found in all the samples. ${ }^{40}$ The extent of the blue shifts in the absorption edges for the CdS NPs has been found to be surfactant dependent. In addition, the UV-vis spectra of the CdS NPs display characteristic shoulders in the $390-410 \mathrm{~nm}$ region due to $1 \mathrm{~S}-1 \mathrm{~S}$ excitonic transitions. ${ }^{41}$ The positions and shapes of these absorption shoulders have also been found to be surfactant dependent. Moreover, the baseline absorbance of the UV-vis spectra can also be taken as indicative of the presence of some bigger NP agglomerates in the sample. ${ }^{42}$ The presence of some large agglomerates is also evident from the higher baseline absorbance of the bulk CdS and the DTAB stabilized NPs, which settled at the bottom of the flask after agglomeration. A comparative account of the effectiveness of different surfactants in the synthesis of CdS NPs in terms of various UV-vis spectral features has been summarized in Table 2. The sizes of the semiconductor NPs can be correlated to the position of the absorption onset, which can be easily determined from the absorption spectra. The optical band gap of the CdS nanodispersions has been determined using the Tauc relation ${ }^{43}$ given in eqn (1).

$$
(\varepsilon h v)=C\left(h v-E_{\mathrm{g}}\right)^{m}
$$

where $C$ is a constant, $\varepsilon$ is the molar extinction coefficient, which can be obtained from the measured absorption spectra using the Beer-Lambert law, $E_{\mathrm{g}}$ is the average band gap of the material and $m$ depends on the type of transition. For $m=1 / 2$, the $E_{\mathrm{g}}$ in eqn (1) is a direct allowed band gap. The average band gap was estimated from the intercept of the linear portion of the $(\varepsilon h v)^{2} v s$. $h v$ plots on the $h v$ axis, as shown in

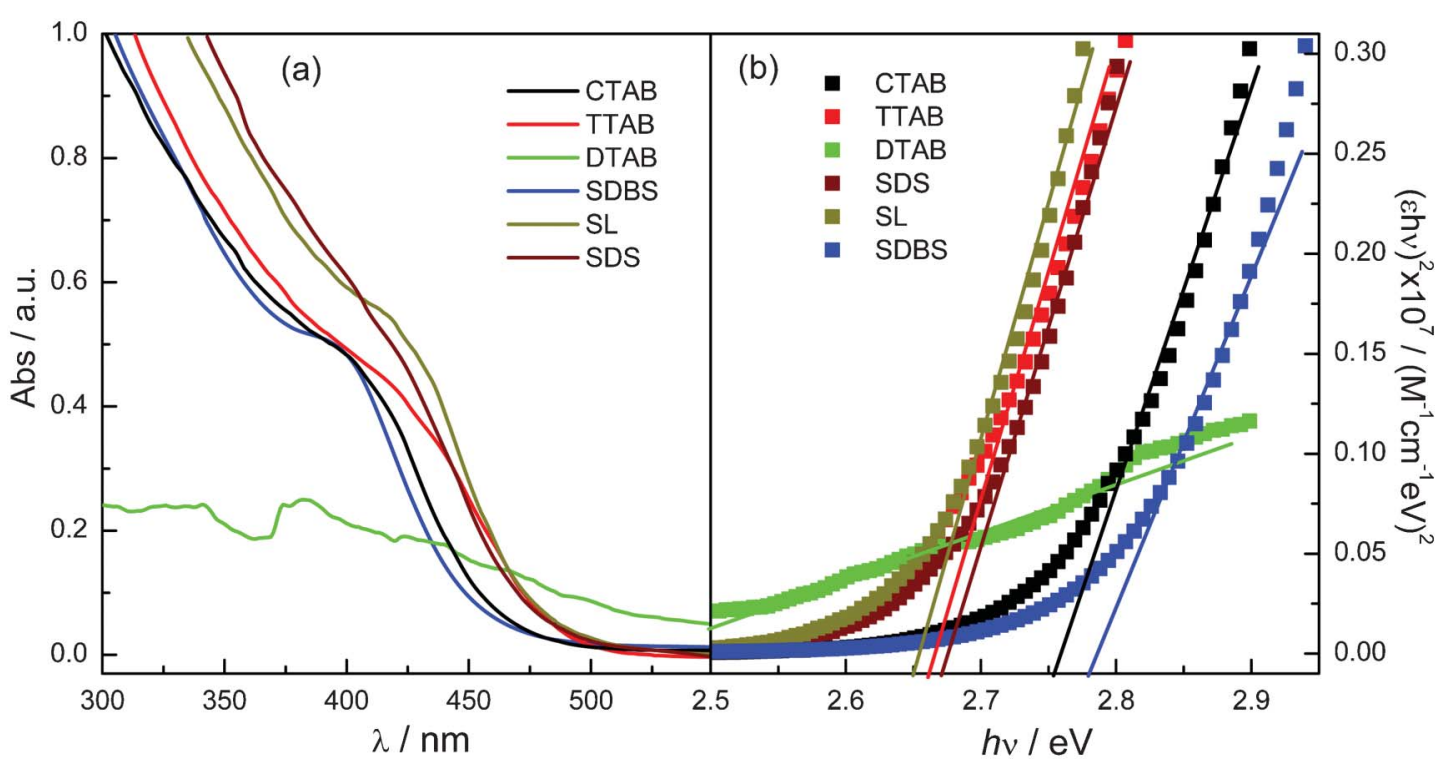

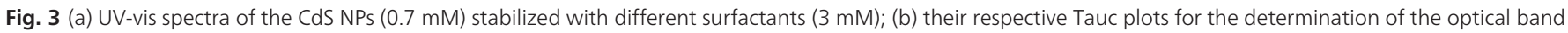
gap. 
Table 2 UV-vis spectral features, optical band gap and sizes of the surfactant stabilized CdS NPS

\begin{tabular}{llllll}
\hline $\begin{array}{l}\text { Surfactant }] \\
(3 \mathrm{mM})\end{array}$ & $\begin{array}{l}\lambda_{\text {edge }}(\mathrm{nm}) \\
\pm 1 \mathrm{~nm})\end{array}$ & $\begin{array}{l}E_{\mathrm{g}}(\mathrm{eV}) \\
\pm 0.02)\end{array}$ & $\begin{array}{l}d_{\text {abs }}(\mathrm{nm}) \\
\pm 0.1)\end{array}$ & $\begin{array}{l}\text { Baseline of absorption } \\
\text { spectral at 350 } \mathrm{nm}( \pm 0.002)\end{array}$ & $\begin{array}{l}\text { Slope of the linear region of the UV-spectra between } \\
\text { the absorption edge and shoulder, } \mathrm{M}_{\mathrm{ES}}(-\mathrm{ve})\left(\times 10^{-2}\right)\end{array}$ \\
\hline CTAB & 479 & 2.76 & 2.70 & 0.014 & $6.135 \pm 0.05$ \\
CTAC & 490 & 2.84 & 2.40 & 0.002 & $6.450 \pm 0.04$ \\
CPyC & 463 & 2.89 & 2.20 & 0.010 & $6.803 \pm 0.04$ \\
TTAB & 481 & 2.66 & 3.10 & 0.003 & $6.041 \pm 0.03$ \\
DTAB & 525 & $>2.50$ & 32.5 & 0.050 & $1.332 \pm 0.01$ \\
SL & 489 & 2.65 & 3.15 & 0.004 & $9.862 \pm 0.05$ \\
SDS & 475 & 2.68 & 3.00 & 0.010 & $7.581 \pm 0.01$ \\
SDBS & 470 & 2.78 & 2.60 & 0.002 & \\
& & & & &
\end{tabular}

Fig. 3(b). The sizes of the NPs were then calculated from the band gap values using the effective mass approximation (EMA). ${ }^{44}$ The values of the effective mass of an electron $\left(m_{\mathrm{e}}\right)$ and that of a hole $\left(m_{\mathrm{h}}\right)$ used in the NPs size calculations were $0.2 m_{\mathrm{o}}$ and $0.8 m_{\mathrm{o}}$, respectively, $m_{\mathrm{o}}$ being the rest mass of an electron. Though the EMA model includes certain approximations, it is widely used in the literature and is sufficient for our comparative study. The optical band gap values and average sizes of the CdS NPs in different surfactants calculated using the EMA are given in Table 2. On comparing the cationic surfactants with different chain lengths, smaller size NPs have been produced in the presence of surfactants with the longest hydrophobic chain length and the size increases on decreasing the hydrophobic chain length. The DTAB stabilized CdS NPs have been found to be least stable and precipitated within half an hour of their formation. These differences can be attributed to the stronger adsorption tendencies of long chain surfactants onto the particle surface due to their increased hydrophobicity. ${ }^{45}$ On the other hand, even the anionic surfactants with a small chain length $\left(\mathrm{C}_{12}\right)$ are able to stabilize the CdS NPs at smaller sizes, depending upon their head group functionalities, though the NP sizes are still greater than those stabilized in CTAB. This might be due to the larger polarizability of the anionic head groups and a correspondingly stronger interaction with the surface of the CdS NPs. Among anionic surfactants with the same chain length, the head group functionality has again played a prominent role by stabilizing the smallest size CdS NPs in SDBS. On the basis of the above discussion, it can be concluded that the increased hydrophobicity as well as a head group with polarizable functionality, such as an aromatic ring, in the surfactant tends to provide better adsorption over the NP's surface to stabilize them at smaller sizes.

It is important to mention that the NP sizes calculated on the basis of the EMA give an average particle size because of some polydispersity in the samples, as evident from the DLS measurements. However, the DLS analysis does not provide the true polydispersity index in each sample of the CdS NPs (core semiconductor material only) because it measures the hydrodynamic size of the NPs, taking into account the adsorbed surfactant layer/surfactant aggregates as well as the hydration sphere of the counterions.

Therefore, the variation in the mode of the surfactant adsorption/aggregation over the surface of the CdS NPs would also affect the particle size distribution analyses. The actual distribution of the CdS NPs core, excluding the surfactant layer, is expected to be different. The changing size distributions of CdS and CdTe NPs have been correlated to the difference in the position of the absorption edge and the peak i.e., $\Delta \lambda=\lambda_{\text {onset }}-\lambda_{\text {peak. }}{ }^{46}$ Moreover, Searson and co-workers ${ }^{47}$ have also demonstrated that the particle size distribution of the semiconductor NPs is related to the local slope of the absorption edge and the NPs have a broader absorption shoulder than a single crystal due to the particle size distributions. Since the UV-vis absorption spectroscopy takes into account the light absorption characteristics of the semiconductor material only, the information derived about the particles size distributions on the basis of this technique can be taken as the true distribution of the NPs. In spite of certain quantum mechanical assumptions during the transformation of the spectral characteristics into particle size distribution profiles, the method is reliable enough for comparative analyses of different systems under similar conditions. A comparison of the size distributions of the CdS NPs stabilized with different surfactants has been obtained from a recently developed spectral slope $\left(\mathrm{M}_{\mathrm{ES}}\right)$ method. ${ }^{48}$ The results are in presented in Table 2. As expected, the size distribution trends for the CdS NPs are different from those estimated on the basis of the polydispersity index (PDI) due to differences in the aggregation of the surfactant over the NPs surfaces.

\subsection{Kinetics of NP formation}

The UV-vis absorbance of semiconductor NPs is directly proportional to the number of absorbing particles (assuming a constant NPs size), i.e., the concentration of NPs formed during the process. Therefore, the process of particle formation, growth and stability can be easily monitored using UVvisible spectroscopy by measuring the changing spectral features and absorbance changes as a function of the time elapsed after the addition of cadmium acetate. Ma et $a{ }^{49}{ }^{49}$ have evaluated the kinetics of aggregation of mononucleotide stabilized CdS NPs in aqueous solution by monitoring the loss of absorbance at $430 \mathrm{~nm}$ as a function of time. The effect of the surfactant structure on the kinetics of the nucleation and growth of the CdS NPs in an aqueous micellar solution has been measured by monitoring the absorbance changes in between the absorption edge and absorption shoulders, where most of the NPs contribute to the spectrum. The time 
dependent spectral absorbance has been monitored at $395 \mathrm{~nm}$ for $\mathrm{CTAB}$ and TTAB and at $450 \mathrm{~nm}$ for the anionic surfactants and DTAB. The two wavelengths selected correspond to the vicinity of the shoulder in the absorption spectra where a maximum shift has been noticed, as discussed later. The time dependent absorption profiles of the CdS NPs stabilized with different surfactants are presented in Fig. 4. The concentrations of the surfactants $(3 \mathrm{mM})$ as well as that of the $\mathrm{Cd}^{2+}$ ions (0.7 $\mathrm{mM})$ were kept the same in each system for a better comparison of the variations in the time dependent UV-visible spectra and absorbance profiles.

The CdS NPs in all the surfactants except TTAB and DTAB display similar absorbance profiles with an initial steep increase approaching a plateau region of constant absorbance.
The initial absorbance values indicate that the nucleation was instantaneous and most of the nuclei formed within a mixing time of 30-35 s before starting the absorbance measurements. The much steeper uptrend in the first few minutes and the time taken to reach the plateau significantly depend upon surfactant-NP interactions. In TTAB, the absorbance displays a steep rise within the first $10 \mathrm{~min}$ and then starts decreasing with a small plateau at the maximum absorbance due to the disappearance of some nucleated particles which aggregated into bigger ones. However, the CdS NPs formed in DTAB have been found to be stable for the initial $20 \mathrm{~min}$ only and thereafter display a sharp prominent decrease in the absorbance due to their precipitation to the bottom of the flask, which was also evident from a visual inspection of the sample.

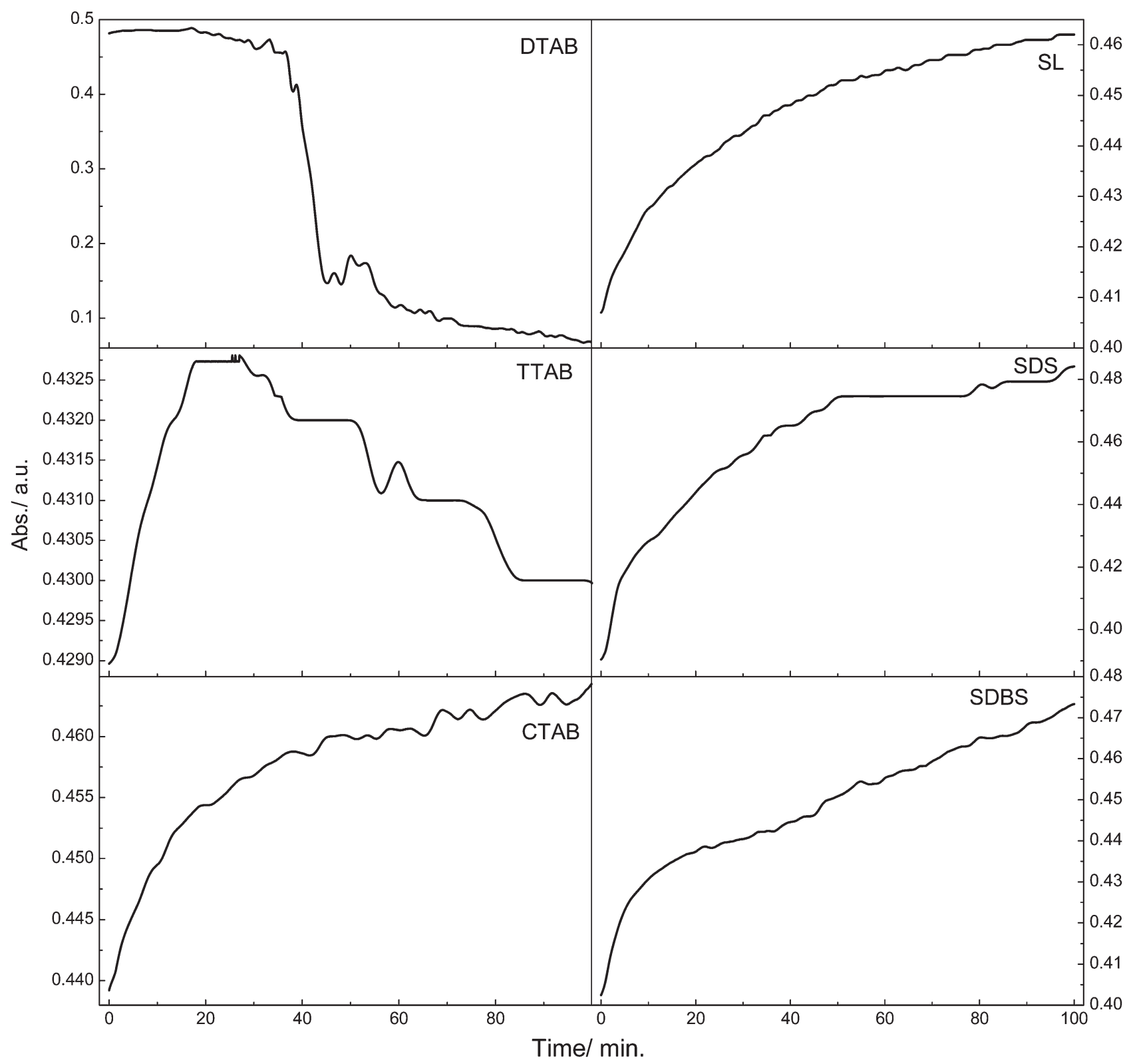

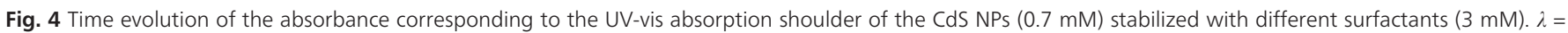
$395 \mathrm{~nm}$ (for CTAB and TTAB), $\lambda=450 \mathrm{~nm}$ (for the anionic surfactants and DTAB). 
Table 3 First-order rate constants obtained from fiting eqn (2) to the time evolution of the absorbance during the nucleation and growth of the particles in the presence of different surfactants

\begin{tabular}{lll}
\hline Surfactant $](3 \mathrm{mM})$ & $A_{\infty}$ (a.u.) & $k\left(\mathrm{~min}^{-1} \times 10^{-2}\right)$ \\
\hline CTAB & 0.460 & $15.79 \pm 0.31$ \\
SL & 0.465 & $8.03 \pm 0.05$ \\
SDS & 0.485 & $14.93 \pm 0.23$ \\
SDBS & 0.462 & $5.64 \pm 0.06$
\end{tabular}

Since the surfactant concentration was kept constant at $3 \mathrm{mM}$ and sodium sulfide was taken in excess, the formation of CdS NPs in all the surfactants except TTAB and DTAB has been assumed to follow pseudo first-order kinetics. The absorbance profiles in Fig. 4 are equivalent to fits by a first-order rate eqn. ${ }^{50,29}$

$$
A_{t}=A_{\infty}\left(1-e^{-k t}\right)
$$

where $A_{t}$ is the absorbance at time $t, A_{\infty}$ is the maximum absorbance attained at a plateau after one hour and $k$ is the first order rate constant. The results of these fits are summarized in Table 3. By changing the head group structure, an interesting behavior has been observed in the anionic surfactants where the rate constant in SDS and SL has been found to be 2.7 and 1.5 times that of SDBS, respectively.

The influence of changing the head group has been found to support our earlier observation of a 3.7 fold increase in the rate constant by changing the head group from pyridinium to trimethylammonium. ${ }^{29}$ The results reveal that the larger and more polarizable head group size probably inhibits the process of particle formation/growth due to different kinds of interactions with the CdS NPs. Additionally, the changing spectral features with time have also been visualized by recording a set of ten spectra at 2 min intervals in each of the surfactants and the results are presented in Fig. S4 (ESI $\dagger$ ). It can be interpreted from Fig. S4 (ESI†) that along with an increase in the absorbance, the position of the UV-vis spectra of the CdS NPs has also been red shifted with time to reach a limiting value that depends upon the type of surfactant. This can be attributed to the growth of small nucleated NPs to reach a stable critical size along with the formation of some new nuclei. It is interesting to notice that in the case of the CdS NPs, the red shift is not observed in the whole spectra but only in a particular portion, with almost no change in the absorption edges. Also, the portion of the CdS NPs spectra which has been red shifted and the extant of the shift has been found to be surfactant dependent. An almost negligible spectral shift in TTAB reveals that the NPs have immediately grown to a certain stable size. On the other hand, the DTAB could not stabilize the CdS NPs and the spectrum shows a downward shift as the particles start precipitating down. In anionic surfactants, the spectral features also display a shift in a particular wavelength range without significantly affecting the band edge position.

Recently, it has been argued that the growth of the NPs may result from coalescence, Ostwald ripening and the reactions of ions with nuclei. ${ }^{51}$ In the present case, neither the decrease in the absorbance (except TTAB and DTAB) nor any prominent red shift in the band edge position has been observed. Therefore, it is believed that the steady growth of the CdS NPs is through reactions of ions with nuclei. This leads to certain structural transitions in the CdS NPs within the first hour without significantly affecting the average particle size. ${ }^{52}$ The varying size distributions of the CdS NP at different growth times have also been correlated to the changing spectral slopes $\left(\mathrm{M}_{\mathrm{ES}}\right)$ for each set of time evolution UV-vis spectra presented in Fig. S4.† The estimated values are depicted in Fig. 5. On the basis of similar studies ${ }^{53}$ upon the temporal evolution of CdS and CdTe NCs, three growth regimes have been discerned with respect to the polydispersity of the nanodispersions. However, we have identified only two regions with respect to the polydispersity in growing CdS NPs, i.e. the region of the initial increase followed by the region of the steady decrease in the $\mathbf{M}_{\mathrm{ES}}$ values. In the initial increasing of $\mathbf{M}_{\mathrm{ES}}$, there is a 'focusing' of the size distribution in all the surfactants except DTAB. The extent of the focusing seems to be surfactant

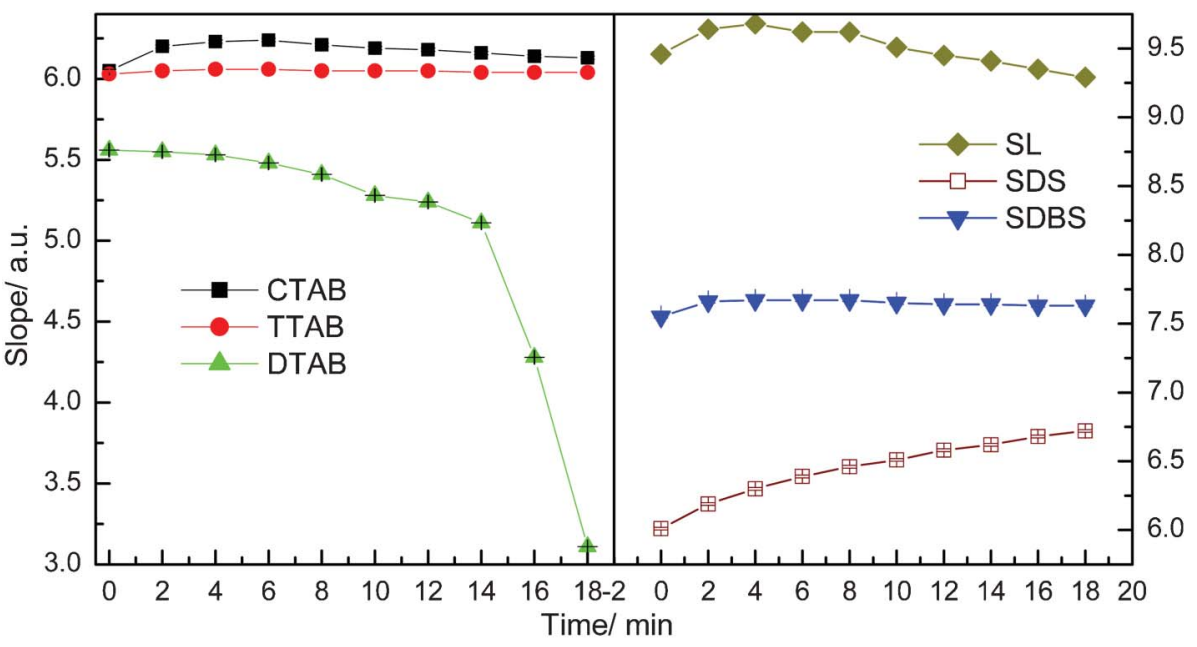

Fig. 5 Change in the UV-vis spectral slope of the CdS NPs with growth time in various surfactants. 
dependent and this region has been evidently absent in the NPs stabilized with DTAB. This result stands in agreement with the earlier report ${ }^{54}$ on the synthesis of thioglycolic acid (TGA)-capped CdTe NCs, where no focusing effect was observed. Thereafter, the event of decreasing $\mathbf{M}_{\mathrm{ES}}$ has been described as 'defocusing' or Ostwald ripening by Peng et al. ${ }^{55}$

From the prominent 'defocusing' observed for DTAB stabilized CdS NPs it is evident that this event can also result from the aggregation of the NPs, which is quite different from Ostwald ripening. Defocusing of the particle sizes has not been observed for the NPs in SDS, whereas this event is present to a small extent in SDBS. The third stage, termed as the 'equilibrium' stage, in which the size distribution does not change with time, is virtually absent in all the samples. Theoretical aspects of 'focusing' or 'defocusing' of the particle size distribution in an ensemble of growing NPs during Ostwald ripening has also been described by Talapin et al. ${ }^{56}$

\subsection{Long time stability and UV-irradiation}

Apart from monitoring the formation of the CdS NPs for initially one hour, it would be of great importance to access the prevalent changes in the NPs after a long period of storage in aqueous dispersions. This aspect of the CdS NPs has been analyzed by comparing their UV-vis spectra, recorded after keeping them under normal room temperature conditions for $24 \mathrm{~h}$. The spectral results are presented in Fig. 6 .

All the samples display a decrease in absorbance along with a blue shift in the absorption spectra. A quantitative account of the surfactant dependent changes in UV-vis spectra in terms of the absorbance decrease and blue shift are given in Table 4 . The absorbance decrease can be assigned to two possible processes taking place in the samples. First, the coalescence that involves the reaction of two particles during the growth, leading to a decrease in the total particle density with time, has been suggested ${ }^{51}$ to show an absorbance decrease near the band edge position. Second, the photocorrosion of aqueous CdS NPs, a well known phenomenon, has already been reported $^{57,58}$ to bleach the CdS by daylight in damp air, leading to $\mathrm{CdSO}_{4}$ as product. Since the decrease in absorbance as well as the blue shift has been found to be different in all the samples, it is therefore believed that the photodissolution process has reduced the size of the NPs and the coalescence of the particles has reduced their number densities in the samples. The coalescence process of the NPs has been possibly controlled by the adsorbed surfactant, thereby causing the absorbance differences. The complete dissolution of very small CdS NPs might also have resulted in an absorbance decrease. In principle, the coalescence of two small NPs into a larger one is expected to cause a red shift in the UV-vis spectra.

On the contrary, the photocorrosion of bigger NPs into a smaller one would cause a blue shift. The competition between the two processes has therefore produced different resultant blue shifts in different surfactants, though photocorrosion has the dominant effect. Similar results, although with a very small blue shift, have also been reported by Sato et al. ${ }^{59}$ during their studies on dissolution of CdS NCs in aqueous $\mathrm{NH}_{3}$ for more than a week. The role of the electron/hole in the anodic and cathodic reactions has been discussed by Meissner et al., ${ }^{60}$ explaining the mechanism of sulfate formation during the photocorrosion of CdS NPs. The exceptionally large blue shift in the UV-vis spectra of the CdS NPs stabilized in SDBS justifies the possible role of the $\pi$-electrons of the benzene ring respectively, in the photocorrosion process. Additionally, the maximum absorbance decrease in TTAB and SDS is due to the prominent coalescence of the NPs due to comparatively weak stabilization by these two surfactants, leaving aside DTAB where the NPs have already been precipitated within half an hour of their formation. The photodissolution behaviour of the CdS NPs under UV-light has also been evaluated by comparing the UV-vis absorption spectra. The $24 \mathrm{~h}$ old aqueous dispersions of CdS NPs were further irradiated with UV-light $(254 \mathrm{~nm})$ for $3 \mathrm{~h}$ and the spectra were recorded immediately as well as after a time delay of 1 and $20 \mathrm{~h}$. The results are presented in Fig. 6 and quantitatively in Table 4. An absorbance decrease along with a blue shift similar to those observed in normal room conditions but to a comparatively smaller extent has been observed in all the samples.

These results are in agreement with those reported by Sato et $a .^{59}$ in the photo-etching of CdS NPs with light of different wavelengths greater than $420 \mathrm{~nm}$. On the basis of similar shifts in the absorption spectra, Spahel et $a .^{61}$ have also discussed the photodissolution of CdS NPs into small sized NPs. These changes have been explained on the basis of the decrease in the absolute volume of the NCs. The photocorrosion or photodissolution of the CdS NPs, producing smaller ones, has been evident from the blue shift in the UV-vis spectra along with the decrease in the total density of the NPs. The surfactant dependent varying extent of the spectral blue shifts reveal that the photodissolution process of the CdS NPs depends up on the type of surfactant. Since the photocorrosion and photodissolution processes start at the surface of the NPs directly exposed to the radiations, smaller CdS NPs with a greater surface area are expected to be greatly affected. Moreover, once again the CdS NPs stabilized in SDBS have been found to display a maximum blue shift in their spectra after UV-irradiation when compared to other samples, further supporting the possibility of the $\pi$-electrons participation.

Furthermore, after removing them from UV-irradiation, no significant changes were observed in the UV-vis spectra of the CdS NPs in any of the surfactants, even after $20 \mathrm{~h}$. This signifies that the CdS NPs have now become resistant towards any type of changes. Some researchers ${ }^{34,35}$ have reported the formation of $\mathrm{Cd}(\mathrm{OH})_{2}$ with the slow surface photoreactions of the CdS NPs. It is suggested that the $\mathrm{Cd}(\mathrm{OH})_{2}$ layer generated over the surface of the CdS NPs has passivated the NPs towards further photocorrosion or photodissolution and has attained the limiting size.

\subsection{Mode of surfactant adsorption: FTIR analyses}

The adsorption mode of long alkyl chain cationic and anionic surfactants has been well established ${ }^{62,63}$ by analyzing the shifts in the existing bands and the formation of new bands in the FTIR spectra of the adsorbed surfactants. Fig. S5 and S6 (ESI†) represent comparative FTIR spectra of free and CdS bonded surfactants. For all six surfactants, the $\mathrm{C}-\mathrm{H}$ symmetric and asymmetric stretching vibration modes of the alkyl chain (3000-2800 $\mathrm{cm}^{-1}$ ) appear at the same position in the free and adsorbed state. In cationic surfactants, the peaks in the 1400- 


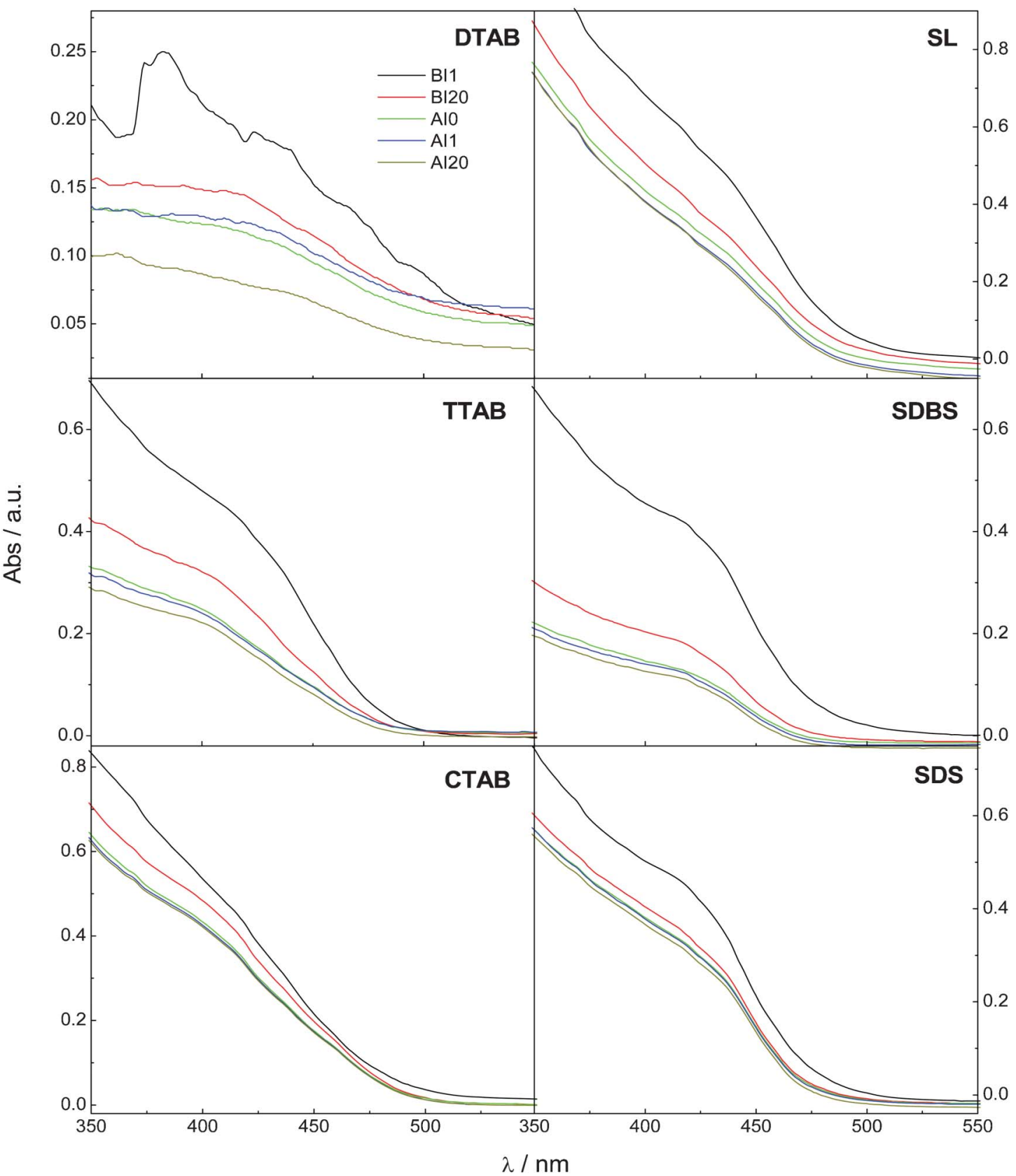

Fig. 6 Effect of the ripening time and UV-light irradiation on the absorption spectra of the CdS NPs ( $0.7 \mathrm{mM})$ prepared in different surfactants ( $3 \mathrm{mM}$ ) after $1 \mathrm{~h}$ of formation (BI1), $20 \mathrm{~h}$ of formation (BI20), immediately after removing them from $3 \mathrm{~h}$ UV-irradiation (AIO) and after 20 h (AI20).

Table 4 Variation in the spectral features after $20 \mathrm{~h}$ ripening of the CdS NPs

\begin{tabular}{llllll} 
Surfactant & \multicolumn{2}{l}{ After $24 \mathrm{~h}$ in normal room temperature conditions } & & \multicolumn{2}{l}{ After $3 \mathrm{~h}$ of UV-irradiation } \\
\cline { 2 - 3 } \cline { 5 - 6 } & Spectral blue shift $(\mathrm{nm})( \pm 0.2)$ & Absorbance decrease $( \pm 0.02)$ & & Spectral blue shift $(\mathrm{nm})( \pm 0.2)$ & Absorbance decrease $( \pm 0.02)$ \\
\hline CTAB & 5.20 & 0.042 & 0.213 & 2.60 & 0.040 \\
TTAB & 4.80 & 0.050 & - & 0.061 \\
DTAB & - & 0.174 & 2.50 & 0.010 \\
SL & 3.10 & 0.239 & 2.70 & 0.060 \\
SDS & 6.80 & 0.10 & 4.10 & 0.027 \\
SDBS & 12.9 & & & 0.055
\end{tabular}




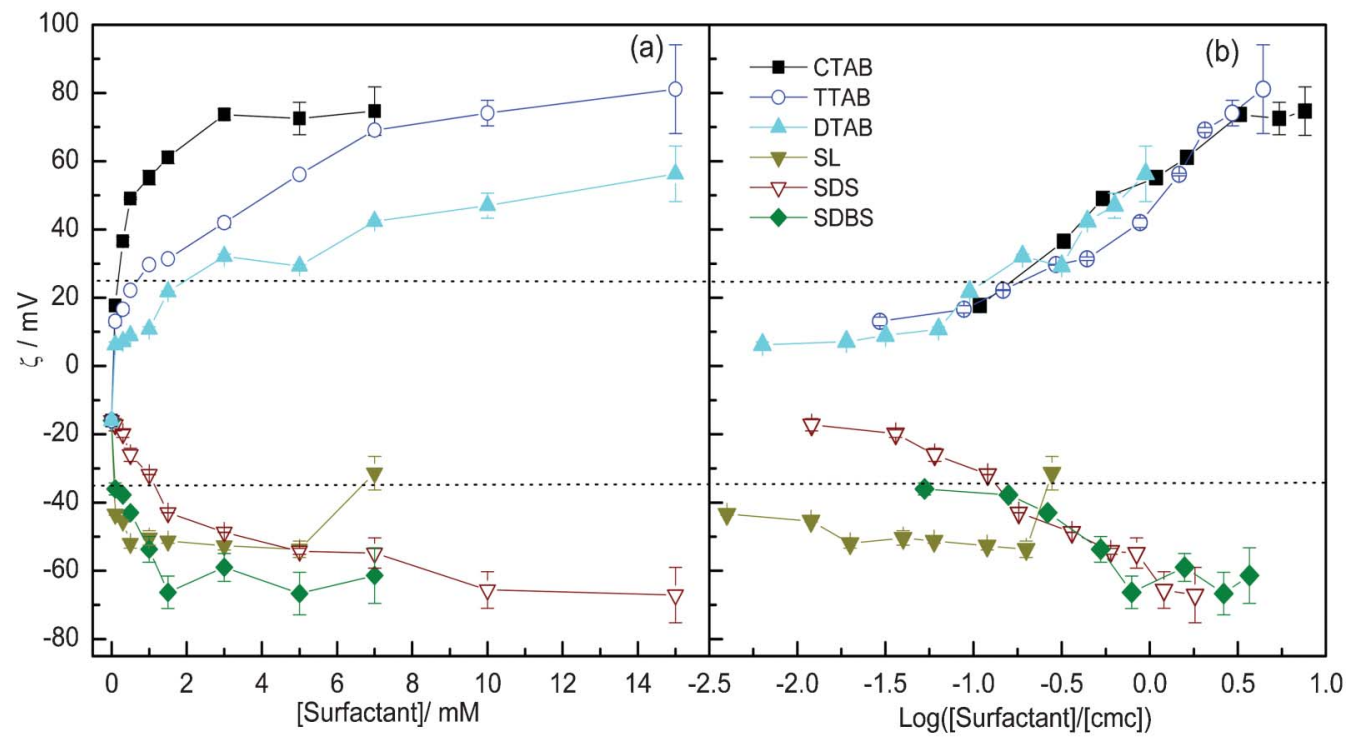

Fig. 7 Variation in the zeta potential ( $\xi$ ) of the surfactant stabilized CdS NPs (0.7 mM) for various surfactants as a function of (a) the surfactant concentration (b) the surfactant concentration normalized with respect to the corresponding cmc's (critical micelle concentration) (with the $30 \mathrm{mV}$ stability line indicated as dotted lines).

$1500 \mathrm{~cm}^{-1}$ range have been assigned to $\mathrm{C}-\mathrm{H}$ scissoring vibrations of the $-\mathrm{N}-\mathrm{CH}_{3}$ moiety and the bands at 1072 and $965 \mathrm{~cm}^{-1}$ are due to $\mathrm{C}-\mathrm{N}$ stretching. ${ }^{64,65}$ In $\mathrm{SL}$, the $-\mathrm{C}=\mathrm{O}$ stretching peak appears at $1705 \mathrm{~cm}^{-1}$ and the peak at 1426 $\mathrm{cm}^{-1}$ has been assigned to a complex structure of the carboxylate head group of the surfactant over the NP surface. ${ }^{63,66}$ In SDS, the peaks at 1080 and $968 \mathrm{~cm}^{-1}$ have been assigned to S-O stretching vibrations and weakly bonded sulfate. ${ }^{67}$ All these peaks, due to the head group moieties of the surfactants, have either been shifted or completely suppressed in the presence of CdS particles. The adsorption of SDBS over the NPs surface has been evidenced by the suppression and shift in the position of the peaks at 3057 $\mathrm{cm}^{-1}$ (aromatic $=\mathrm{C}-\mathrm{H}$ starching), $1468 \mathrm{~cm}^{-1}$ and $1417 \mathrm{~cm}^{-1}$ (aromatic C-C stretching vibrations. The peak at $1124 \mathrm{~cm}^{-1}$ due to sulfonate adsorption has also been shifted. ${ }^{67}$ The above FTIR analyses therefore indicate that the surfactants have been adsorbed over the NPs surface through their head groups, leaving the hydrocarbon chain free.

\subsection{Zeta potential measurements}

The zeta potential is a physical property of any particle in a suspension. ${ }^{68}$ It is also an aid in predicting the long-term stability of a suspension. The magnitude of the zeta potential gives an indication of the potential stability of the colloidal system. If all the particles in the suspension have a large negative or positive zeta potential then they will tend to repel each other and there will be no tendency for the particles to come together. However, if the particles have low zeta potential values then there will be no force to prevent the particles coming together and flocculating. The general dividing line between stable and unstable suspensions is generally taken at either +30 or $-30 \mathrm{mV}$. Particles with zeta potentials more positive than $+30 \mathrm{mV}$ or more negative than $-30 \mathrm{mV}$ are normally considered stable. The stabilizing tendencies of various surfactants towards CdS NPs in aqueous media have been assessed by comparing the zeta potential values. Fig. 7 presents the variation in the zeta potential of the CdS NPs $(0.7 \mathrm{mM})$ as a function of the surfactant concentrations used for the stabilization of the NPs. The zeta potential of aqueous CdS dispersions without any added surfactant has been measured to be $-16 \pm 2 \mathrm{mV}$ due to the presence of excess $\mathrm{S}^{2-}$ and $\mathrm{HS}^{-}$ions near the surface, forming an electrical double layer. However, the magnitude of the zeta potential of the CdS NPs is given solely by the nature and concentration of the surfactants. The zeta potential values show a slow increase after a particular surfactant concentration characteristic for that surfactant. In SL and SDBS, the zeta potential even starts decreasing after attaining a peak value. These observations reveal that a definite amount of each surfactant, depending upon their molecular structure, is sufficient to stabilize the CdS NPs and that excess surfactant is either not involved in the stabilization or even shows a destabilizing effect. A larger deviation from the mean zeta potential value at high surfactant concentrations probably arises from the formation of free micelles by excess surfactant after the NPs surface coverage is complete. For a given surfactant concentration, the zeta potential values and hence the stability of the NPs increases with the hydrocarbon chain length of the cationic surfactants. The DTAB stabilized CdS NPs have been found to be least stable at lower surfactant concentrations and flocculate within $30 \mathrm{~min}$ of their formation.

However, the NPs are stable above $7 \mathrm{mM}$ DTAB concentrations, though not for a long time, and show a high standard deviation in their zeta potential. The extra methylene groups have possibly induced greater hydrophobicity to the cationic surfactants, which provides a better capping ability and a stronger liquid-solid interfacial adsorption. ${ }^{69}$ On the other hand, the CdS NPs stabilized with anionic surfactants with 
similar $\mathrm{C}_{12}$ hydrocarbon chains have been shown to have good long time stability even at lower concentrations, depending upon their head group structures. This significant difference in stabilization tendency suggests that in addition to the increased hydrophobicity of the cationic surfactants, the stronger interactions of the anionic head groups with the NPs surface are also responsible in the NP stabilization process. SDS has been able to stabilize the CdS NPs above concentrations of $1.5 \mathrm{mM}$, whereas SL and SDBS stabilize the NPs at still lower concentrations i.e. above $0.1 \mathrm{mM}$. The approximate amount of surfactant required for the full surface coverage of the NPs has also been estimated by assuming the particles to be spherical. Since the head group area of the surfactants used in the present study ranges between 0.32-0.65 $\mathrm{nm}^{2}$, the calculations (Appendix A) reveal that approximately 0.38-0.77 $\mathrm{mM}$ of surfactant has been directly involved in the surface coverage of the CdS NPs. An excess of the surfactant is needed to prevent the agglomeration of the NPs by keeping them sufficiently apart. In spite of same head group areas of CTAB, TTAB and DTAB, these surfactants display significantly different stabilization tendencies, as assessed from the zeta potential values. Therefore, the varying aggregation behavior (in terms of $\mathrm{cmc}$ ) can be held responsible for the varying stabilization of the NPs, though their precise contribution can not be evaluated since the cmc values have been greatly influenced by the presence of inorganic ions. ${ }^{64}$

More precise information regarding the stabilization of the CdS NPs has been deduced by comparing the zeta potential values for the different surfactants in units of their $\mathrm{cmc}$ (Fig. 7(b)). By analyzing the cationic and anionic surfactants separately, one can observe that the data in each set look very similar and fall on a master curve, where the point of zero charge occurs in a symmetric fashion. This indicates that in the present systems, the micelle formation of the pure surfactants plays an important role but the zeta potentials are moved into regimes of colloidal stability already for concentrations in the range of $20-60 \%$ of the cmc. Interestingly, the anionic surfactants, except SL, have effectively lowered the zeta potential when compared to that raised by the corresponding cationic surfactants. This has been assigned to specific interactions of the head groups that are apparently more pronounced for the anionic case compared to the cationic one. The negative zeta potential of bare $\mathrm{ZnS}$ suspensions in aqueous solution changes to positive and negative in the presence of cationic and anionic surfactants, respectively. On the basis of the transformations in the zeta potential sign and FTIR analyses, a nearly bilayer surfactant structure over the NP's surface is believed to be the most probable adsorption mode.

\subsection{PL emission}

The conjugation of semiconductor NPs with surfactants in aqueous media is of particular significance as it allows one to avoid the loss of PL intensity at the stage of ligand exchange and transfer from organic to aqueous media. In semiconductor NPs, the PL is produced due to the recombination of the charge carriers which are generated by light absorption. The recombination can take place from various surface states. If the defect sites are located at the surface of the colloidal NPs,

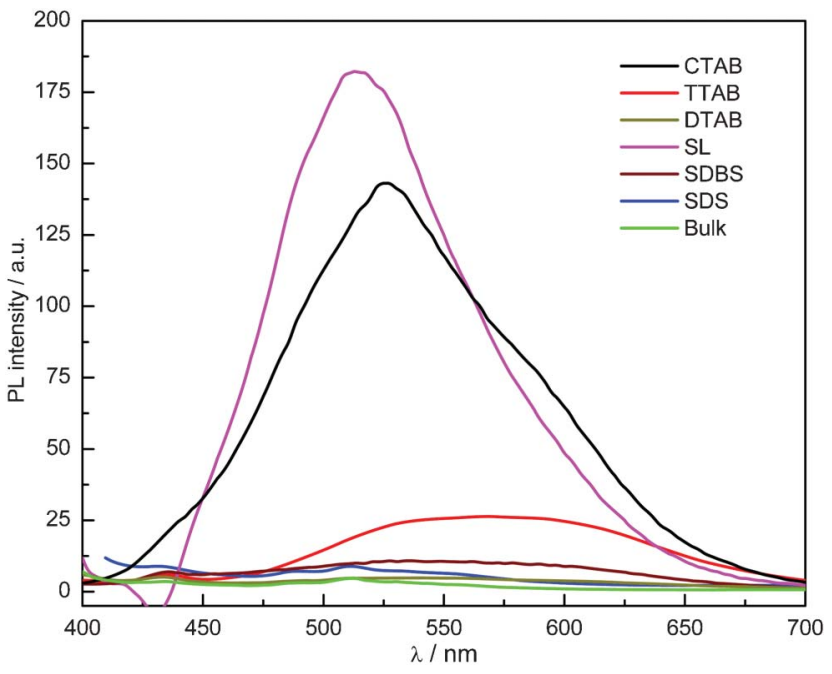

Fig. 8 PL emission spectra of the CdS NPs $(0.7 \mathrm{mM})$ stabilized with different surfactants (3 $\mathrm{mM})$.

there is a chance for these sites to be influenced by the stabilizing material. Therefore, the effect of different surfactants in identical solvent environments on the PL emission behavior of CdS NPs has been analyzed. Fig. 8 presents the comparative PL emission spectra of aqueous suspensions of CdS NPs in the presence of different surfactants. In order to explain the PL emission of the CdS NPs, it is imperative to understand the different relaxation pathways of the electronhole pair formed during the excitation. Because of the high surface area of the NPs, non-radiative relaxation of trap states competes with band edge emission (radiative recombination).

The defects in the crystal lattice, surface structure and particle clustering constitute the main reasons for the formation of temporary surface traps for non-radiative recombination. Apart from band edge PL emission, which is observed in quantum dots with an ideal surface structure, the colloidal CdS NPs generally have a weak broad emission ranging from 500 to $700 \mathrm{~nm}$, attributed to the radiative recombination of charge carriers immobilized in traps of different energies. ${ }^{70}$ It is also important to mention here that the observed emission is not the band edge emission but due to the radiative recombination of charge carriers immobilized in deep-trap states of different energies. ${ }^{61,71}$ The comparatively minor band edge emission at $433 \mathrm{~nm}$ has been overshadowed by the dominant surface-state emission. The position and the intensity of the PL emissions reported in Table 5 are found to be strongly surfactant dependent. A significant increase in the intensity of the PL signal of the CdS NPs in the presence of all the surfactants when compared to bulk CdS (without surfactant) has been clearly observed. It is also noteworthy that the emission peak is quite well defined in all the surfactants except in DTAB and SDS, where featureless spectra nearly identical to that of bulk CdS have been observed. The instability of the CdS NPs in DTAB, leading to their precipitation, is responsible for their negligible bulk-like PL emission. The absence of an emission peak in bulk semiconductor material is due to coupling of the emitting states 
Table $\mathbf{5}$ Various parameters of the surfactant stabilized CdS NPs deduced PL emission spectra

[Surfactant] (3 mM) Position of PL emission FWHM of PL emission maxima $(\mathrm{nm})( \pm 2 \mathrm{~nm})$ peaks $(\mathrm{nm})( \pm 2 \mathrm{~nm})$

\begin{tabular}{lll}
\hline CTAB & 526 & 118 \\
TTAB & 568 & 141 \\
DTAB & 572 & - \\
SL & 514 & 84 \\
SDS & 540 & - \\
SDBS & 538 & 180
\end{tabular}

with lattice vibrations of the extended lattice and is due to a number of dangling bonds which provide traps for nonradiative recombination. ${ }^{72}$

Bol and Meijerink ${ }^{73}$ observed that a higher degree of polymerization can lead to better surface coverage and can reduce the number of dangling bonds on the surface of NPs, thereby increasing the PL emission. The surfactants are therefore believed to eliminate the surface traps caused by dangling bonds and hence help in maintaining better PL emission. Stabilization of the surface defects by the adsorbed surfactants, in a similar manner to those reported in the case of cystein and citrate ligands, ${ }^{34,35}$ can also be responsible for the passivation of non-radiative defect sites.

The low PL emission in DTAB and SDS stabilized CdS NPs indicates that nonradiative recombination of the charge carriers is the dominant process due to weak adsorption of these two surfactants. Since the PL emission intensity is still greater than that of bare CdS, this indicates the elimination of certain defects and the satisfying some dangling bonds. The increased PL emission in all other samples has been found to be related to the effective passivation of surface defects in small particles that otherwise can act as traps for the excitons, producing luminescence comparable to very few and/or inaccessible traps in large crystals. ${ }^{74}$ Hull and coworkers ${ }^{75}$ suggested that the photogenerated electrons in the conduction band are responsible for the PL in CdS NPs. The planar, defectfree surfaces of ionic compound semiconductors do not have surface states in the band gap. ${ }^{76,77}$ However, a surface $\mathrm{S}^{2-}$ vacancy, and also presumably a $\mathrm{Cd}^{2+}$ edge atom, can provide an electron trap. In an analogous fashion, a surface $\mathrm{Cd}^{2+}$ vacancy and/or a $\mathrm{S}^{2-}$ edge atom could provide hole traps. The distribution of electron-transfer is then determined by the density of the trapped holes/electrons and not by the diameter of the cluster. However, NPs with sizes grater than $10 \mathrm{~nm}$ (e.g. in DTAB) do not have prominent PL emission due to the absence of the quantum confinement effect of charge carriers. The PL emission of CdS NPs is affected by the surface charge state and it appears that the luminescence can be a probe of their surface structure. ${ }^{78}$

Moreover, researchers have also reported ${ }^{79}$ that the adsorbing lewis acids draw electrons from the semiconductor bulk to surface states and quench the PL intensity, whereas adsorbing lewis bases enhance the PL intensity. The cationic surfactants (having an ammonium head group), acting as lewis acids towards the NP surface, can stabilize the surface states of the CdS semiconductor to different extents depending upon their structure. CTAB adsorbed on the surface of CdTe NPs has been shown ${ }^{80}$ to stabilize the electrons of electron-hole pairs and decreases the radiative electron-hole recombination, resulting in PL quenching. It has also been suggested that protonated amino groups (i.e. an ammonium moiety) in the amino acids could scavenge electrons from the electron-hole pairs formed as a result of the excitation of CdS NPs and are therefore responsible for quenching the fluorescence. ${ }^{81}$ Also, the surface states of smaller particles can be easily stabilized due to a large percentage of atoms on or near the surface as well as a higher curvature and show less PL emission due to the more efficient transfer of electrons to suitable species adsorbed on the surfaces. On the other hand, it has been demonstrated by Fogg et $a{ }^{82}$ and Veinot et $a{ }^{83}{ }^{83}$ that the coordination of electrondonating compounds, such as an amine and phosphine oxide, significantly improves the PL performance of CdS NPs. Therefore, the anionic surfactants are expected to produce CdS NPs with a better PL emission when compared to cationic surfactants. However, the PL emission of CdS NPs has been found to be in agreement with these results only in one anionic surfactant i.e. SL. On the contrary, very little PL emission is observed for the CdS NPs stabilized in SDS and SDBS. Such a prominent difference in the PL emission can not be justified on the basis of the difference in their particle sizes. It is believed that the nature of the functional group in the surfactant head group and the strength of the surfactant-NPs interactions have dominant roles in modifying the surface states and hence the PL emission. Kepler et $a l^{79}$ have explained the steric factor dependent binding strength of various carbonyl compounds on the surface of CdS(e) NPs as the main contributing factor for PL quenching. They have shown adduct formation to basic chalcogen sites on the CdS(e) surfaces through the carbonyl carbon or oxygen of properly oriented molecules. It is a well established fact that the interactions of the adsorbed surfactants in aqueous media are mainly governed by their increased hydrophobicity and the nature of the head group. Therefore, it is suggested that the bulky head groups in the SDS and SDBS surfactants could not significantly affect the surface states of the CdS NPs due to their weak interactions or orientation when compared to the carboxylate head group of SL.

\section{Experimental section}

\subsection{Materials}

Cadmium acetate $\left(\mathrm{Cd}(\mathrm{OAc})_{2}, 99 \%\right)$ and sodium sulfide $\left(\mathrm{Na}_{2} \mathrm{~S} \cdot x \mathrm{H}_{2} \mathrm{O}, 58 \%\right.$ assay) were purchased from $\mathrm{CDH}$, India. CTAB (99\%), tetredecyltrimethylammonium bromide (TTAB; 99\%), dodecyltrimethylammonium bromide (DTAB; 99\%), sodium lauarte (SL; > 97\%) and sodiumdodecylbenzene sulfonate (SDBS; 99\%) were purchased from Sigma Aldrich and SDS (98\%) was purchased from Merck. All the chemicals were used as received without further purification. The water used in the preparation of the solutions was double distilled. Table S1 (ESI $\dagger$ ) lists the cmc values of the surfactants determined in the lab using the conductivity method. The cmc values are in close agreement with the literature values. 


\subsection{Synthesis of the surfactant stabilized CdS NPs}

The synthesis of the CdS NPs was performed using the chemical precipitation method ${ }^{29}$ keeping the $\mathrm{Cd}(\mathrm{OAc})_{2}: \mathrm{Na}_{2} \mathrm{~S}$ molar ratio as $1: 2$ at room temperature. Briefly, a certain volume of an aqueous $\mathrm{Na}_{2} \mathrm{~S}$ solution was added to $10 \mathrm{ml}$ of an aqueous surfactant solution so as to make the final concentration $1.4 \mathrm{mM}$. The solution was kept at room temperature for half an hour for equilibration and then an aqueous $\mathrm{Cd}(\mathrm{OAc})_{2}$ solution was added into the above solution with gentle shaking to obtain a final $\mathrm{Cd}^{2+}$ concentration of $0.7 \mathrm{mM}$. The appearance of light yellow colorations indicates the formation of CdS NPs.

\subsection{Characterizations}

The CdS NPs were characterized using a Hitachi (H-7500) transmission electron microscope (TEM) operating at $100 \mathrm{kV}$. The samples for the TEM studies were prepared by placing a drop of the CdS NPs dispersed in an aqueous surfactant solution onto a carbon coated $\mathrm{Cu}$ grid and the solvent was evaporated at room temperature. A JEOL (JSM-6100) scanning electron microscope (SEM) operating at $20 \mathrm{kV}$ was used to establish the surface morphology of the powdered samples. XRD studies were carried out using a Panalytical, D/Max-2500 $\mathrm{X}$-Ray Diffractometer equipped with $\mathrm{Cu}-\mathrm{K} \alpha$ radiation $(\lambda=$ $1.5418 \mathrm{~A}^{\circ}$ ) and employing a scanning rate of $0.02^{\circ} \mathrm{s}^{-1}$. The FTIR spectra of the CdS NPs were recorded with a Perkin Elmer RX-1 spectrophotometer in the $3200-900 \mathrm{~cm}^{-1}$ frequency range using $\mathrm{NaCl}$ plates. The samples were prepared by making a thick paste of a washed and dried sample of the NPs in $\mathrm{CCl}_{4}$.

The absorbance and PL spectra were recorded with a Jasco$530 \mathrm{~V}$ spectrophotometer and a Perkin Elmer LS55 luminescence spectrometer, respectively using a quartz cuvette with a $1 \mathrm{~cm}$ path length. The PL excitation wavelength was $320 \mathrm{~nm}$. For the time evolution absorption and PL measurements, the particles were nucleated by quickly adding an aqueous $\mathrm{Cd}(\mathrm{OAc})_{2}$ solution into the aqueous surfactant solution (3 $\mathrm{mM}$ ) containing sodium sulfide. The resultant solution was then immediately transferred into a quartz cuvette for the absorption measurements. The mixing time was about 40-45 s before starting the absorption spectral measurements. The zeta potential of the NPs was determined from their electrophoretic mobilities according to Smoluchowski's approximation using a Malvern Zetasizer NanoZS (Zen 3600) at $25{ }^{\circ} \mathrm{C}$ using a folded capillary cell (DTS 1060).

The DLS experiments were performed using an ALV/CGS-3 Compact Goniometer System with a He-Ne laser $(\lambda=632.8$ $\mathrm{nm}$ ) equipped with 2 Avalanche Photo Diodes (APD) for pseudo cross-correlation. All the measurements were performed at a scattering angle equal to $90^{\circ}$ for the different NPs suspensions. A sample cell was set in the toluene bath for index matching with the quartz cell and the temperature was maintained at $25{ }^{\circ} \mathrm{C}$ in the toluene bath. The data were analysed with the program ALV-7004 version 3.0, which does an inverse Laplace transformation using a constrained regularization method and thereby yielding a relaxation spectrum of the autocorrelation function.

\section{Conclusions}

Detailed investigations on the evolution of the optical properties of CdS NPs stabilized in different surfactants have provided a better insight about the surfactant structure dependence of the properties. All the cationic and anionic surfactants, except DTAB, used as stabilizing agents have proven to be ideal to obtain adequately stabilized CdS NPs in aqueous media with a small size range. The surfactant head group and hydrophobic chain length do affect the size as well as the optical and PL emission properties in aqueous media. Under identical conditions, the efficiency of the surfactants in controlling the size and surface states of the CdS NPs have been found to depend upon the surfactant adsorption and thus CdS NPs with a smaller size have been stabilized by the surfactants with the longest hydrocarbon chain due to increased hydrophobicity. Zeta potential measurements reveal that the intermediates surfactant concentrations, irrespective of the cmc, are needed to stabilize the CdS NPs. When extracted from aqueous solution, the NPs average the same primary crystallite sizes irrespective of the nature of the surfactants. The surfactants serve to modulate the emission properties of the particles to different extents. Among the cationic surfactants, CTAB is believed to be the most efficient in stabilizing the surface of the CdS NPs with an excellent PL emission. On the other hand, SL has been proven to passivate the CdS NPs by blocking the non-radiative recombination channels of the surface defect states. The exceptionally high rate constant, $k$, for the growth of the CdS NPs in CTAC and the reverse trend in the time dependent PL intensity profile open new avenues to extend the study to other semiconductor NPs in order to explore the origin of these changes.

\section{Appendix A: Determination of the surfactant required for the NPs surface coverage}

No. of surfactant molecules required to cover the surface of the NPs $=$ (total no. of NPs in the solution $\times$ the surface area of one NP)/head group area of one surfactant molecule.

Surface area of one spherical NP $=4 \pi r^{2}$

Total no. of NPs = total no. of CdS units/no. of CdS units in one particle.

Total no. of CdS units $=\left[\mathrm{Cd}^{2+}\right] \times N_{\mathrm{A}}$; where $N_{\mathrm{A}}$ is avogadro's number.

No. of CdS units in one particle = agglomeration number of CdS NPs $=84.2 r^{3}$; here $r$ is the radius of the NPs in $\mathrm{nm}$.

\section{Calculation of the agglomeration number}

The agglomeration number can be calculated by using the following equation [ref. 78]

$$
n=\frac{4 \pi N_{\mathrm{a}} r^{3}}{3 V_{\mathrm{m}}}
$$

where $N_{\mathrm{a}}$ is Avogadro's number, $r$ is the radius of the 
nanoparticle and $V_{\mathrm{m}}$ is the molar volume of CdS. The molar volume $\left(V_{\mathrm{m}}\right)$ is defined as

$$
V_{\mathrm{m}}=\frac{M_{\mathrm{CdS}}}{\rho}
$$

where $M_{\mathrm{CdS}}$ is the Molar mass and $\rho$ is the density of CdS. Putting in the values of $\pi, N_{\mathrm{a}}, M_{\mathrm{CdS}}$ and $\rho=4.82 \mathrm{~g} \mathrm{~cm}^{-3}$, the eqn (1) reduces to

$$
n=84.2 r^{3}
$$

where $r$ is the radius in $\mathrm{nm}$.

\section{Acknowledgements}

Authors are grateful to DST and DAAD for the award of a Project Based Personal Exchange Programme (PPP). Sanjay Kumar is also thankful to CSIR, New Delhi for a Senior Research Fellowship.

\section{References}

1 J. K. Jaiswal, H. Mattoussi, J. M. Mauro and S. M. Simon, Nat. Biotechnol., 2003, 21, 47-51.

2 W. C. W. Chan, D. J. Maxwell, X. H. Gao, R. E. Bailey and M. Y. Han, Curr. Opin. Biotechnol., 2002, 13, 40-46.

3 Y. Li, X. Li, C. Yang and Y. J. Li, J. Mater. Chem., 2003, 13, 2641-2648.

4 A. Henglein, Ber. Bunsen-Ges. Phys. Chem., 1982, 86, 301-305.

5 R. Rossetti, S. Nakahara and L. E. Brus, J. Chem. Phys., 1983, 79, 1086-1088.

6 T. Vossmeyer, L. Katsikas, M. Giersig, I. G. Popovic, K. Diesner, A. Chemseddine, A. Eychmüller and H. Weller, J. Phys. Chem., 1994, 98, 7665-7673.

7 J. Z. J. Zhang, J. Phys. Chem. B, 2000, 104, 7239-7253.

8 G. Shen, J. H. Cho, J. K. Yoo, G.-C. Yi and C. J. J. Lee, J. Phys. Chem. B, 2005, 109, 9294-9298.

9 S. Mandal, D. Rautaray, A. Sanyal and M. Sastry, J. Phys. Chem. B, 2004, 108, 7126-7131.

10 L. Weinhardt, T. Gleim, O. Fuchs, C. Heske, E. Umbach, M. Bar, H.-J. Muffler, C.-H. Fischer, M. C. Lux-Steiner, Y. Zubavichus, T. P. Niesen and F. Karg, Appl. Phys. Lett., 2003, 82, 571-573.

11 C. B. Murray, C. R. Kagan and M. G. Bawendi, Annu. Rev. Mater. Sci., 2000, 30, 545-610.

12 C. J. Barrelet, Y. Wu, D. C. Bell and C. M. Lieber, J. Am. Chem. Soc., 2003, 125, 11498-11499.

13 W. Chen, K. Chen, Q. Peng and Y. Li, Small, 2009, 5, 681-684.

14 Y. Cheng, Y. Wang, F. Bao and D. Chen, J. Phys. Chem. B, 2006, 110, 9448-9451.

15 J. H. Warner and R. D. Tilley, Adv. Mater., 2005, 17, 997-3001.

16 W. T. Yao, S. H. Yu, S. J. Liu, J. P. Chen, X. M. Liu and F. Q. Li, J. Phys. Chem. B, 2006, 110, 11704-11710.

17 P. Wu, Y. Ye, T. Sun, R. Peng, X. Wen, W. Xu, C. Liu and L. Dai, ACS Nano, 2009, 3, 3138-3142.
18 P. C. Wu, Y. Ye, C. Liu, R. M. Ma, T. Sun and L. Dai, J. Mater. Chem., 2009, 19, 7296-7300.

19 M. Chen, Y. Xie, J. Lu, Y. Xiong, S. Zhang, Y. Qian and X. Liu, J. Mater. Chem., 2002, 12, 748-753.

20 C. Ye, G. Meng, Y. Wang, Z. Jiang and L. Zhang, J. Phys. Chem. B, 2002, 106, 10338-10341.

21 A. Pan, R. Liu, Q. Yang, Y. Zhu, G. Yang, B. Zou and K. Chen, J. Phys. Chem. B, 2005, 109, 24268-24272.

22 D. Xu, Z. Liu, J. Liang and Y. Qian, J. Phys. Chem. B, 2005, 109, 14344-14349.

23 R. Thiruvengadathan and O. Regev, Chem. Mater., 2005, 17, 3281-3287.

24 M. Zhang, M. Drechsler and A. H. E. Muller, Chem. Mater., 2004, 16, 537-543.

25 A. Phuruangrat, T. Thongtem and S. Thongtem, Mater. Lett., 2009, 63, 1562-1565.

26 X. Liu, Mater. Chem. Phys., 2005, 91, 212-216.

27 F. Gao, Q. Lu, S. Xie and D. Zhao, Adv. Mater., 2002, 14, 1537-1540.

28 Z. Zhuang, X. Lu, Q. Peng and Y. Li, J. Am. Chem. Soc., 2010, 132, 1819-1821.

29 S. K. Mehta, S. Kumar, S. Chaudhary and K. K. Bhasin, Nanoscale, 2010, 2, 145.

30 G. Pandey and S. Dixit, J. Phys. Chem. C, 2011, 115, 17633-17642.

31 I. Chakraborty, D. Mitra and S. P. Moulik, J. Nanopart. Res., 2005, 7, 227.

32 C. N. R. Rao, A. Govindaraj, F. L. Deepak, N. A. Gunari and M. Nath, Appl. Phys. Lett., 2001, 78, 1853.

33 L. E. Shea-Rohwer and J. E. Martin, J. Lumin., 2007, 127, 499-507.

34 Y. Wang, Z. Tang, M. A. Correa-Duarte, I. Pastoriza-Santos, M. Giersig, N. A. Kotov and M. L. Liz-Marzan, J. Phys. Chem. $B, 2004,108,15461$.

35 Y. Wang, Z. Tang, M. A. Correa-Duarte, L. M. Liz-Marzan and N. A. Kotov, J. Am. Chem. Soc., 2003, 125, 2830.

36 E. Feitosa, M. R. S. Brazolin, R. M. Z. G. Naal, M. P. F. M. D. Lama, J. R. Lopes, W. Loh and M. Vasilescu, J. Colloid Interface Sci., 2006, 299, 883.

37 W. S. Chae, H. W. Shin, E. S. Lee, E. J. Shin, J. S. Jung and Y. R. Kim, J. Phys. Chem. B, 2005, 109, 6204-6209.

38 R. Jenkins and R. L. Snyder, Introduction to X-ray powder diffractions, John Wiley and Sons Inc., Hoboken, New Jersey, 1996, ch. 1, pp. 89-91.

39 P. Scardi and M. Leoni, Acta Crystallogr., Sect. A: Found. Crystallogr., 2002, 58, 190-200.

40 K. T. Yong, Y. Sahoo, M. T. Swihart and P. N. Prasad, J. Phys. Chem. C, 2007, 111, 2447-2458.

41 A. Mews, A. Eychmuller, M. Giersig and H. Weller, J. Phys. Chem., 1994, 98, 934-941.

42 W. G. Becker and A. J. Bard, J. Phys. Chem., 1983, 87, 4888.

43 J. Tauc and A. Menth, J. Non-Cryst. Solids, 1972, 8, 569.

44 Y. Wang, A. Suna, W. Mahler and R. Kasowaki, J. Chem. Phys., 1987, 87, 7315.

45 R. Atkin, V. S. J. Craig, E. J. Wanless and S. Biggs, J. Colloid Interface Sci., 2003, 266, 236.

46 S. Modes and P. Lianos, J. Phys. Chem., 1989, 93, 5854.

47 N. S. Pesika, K. J. Stebe and P. C. Searson, Adv. Mater., 2003, 15, 1289.

48 S. K. Mehta, S. Kumar and M. Gradzielski, J. Colloid Interface Sci., 2011, 360, 497. 
49 N. Ma, J. Yang, K. M. Strewart and S. O. Kelley, Langmuir, 2007, 23, 12783.

50 L. M. Liz-Marzan and I. Lado-Tourino, Langmuir, 1996, 12, 3585 .

51 S. León-Velazquez, R. Irizarry and M. E. Castro-Rosario, J. Phys. Chem. C, 2010, 114, 5839.

52 K. Eichkorn and R. Ahlrichs, Chem. Phys. Lett., 1998, 288, 235.

53 A. Priyam, A. Chatterjee, S. C. Bhattacharya and A. Saha, J. Cryst. Growth, 2007, 304, 416.

54 D. V. Talapin, A. L. Rogach, E. V. Shevchenko, A. Kornowski, M. Hasse and H. Weller, J. Am. Chem. Soc., 2002, 124, 5782.

55 X. Peng, J. Wickham and A. P. Alivisatos, J. Am. Chem. Soc., 1998, 120, 5343.

56 D. V. Talapin, A. L. Rogach, M. Haase and H. Weller, J. Phys. Chem. B, 2001, 105, 12278.

57 D. Richardson, Chem. Nachr., 1886, 54, 297.

58 E. H. Weber, Phys. Status Solidi B, 1969, 36, 175.

59 K. Sato, S. Kojima, S. Hattori, T. Chiba, K. U. Sarson, T. Torimoto, Y. Tachibana and S. Kuwabata, Nanotechnology, 2007, 18, 465702.

60 D. Meissner, C. Benndorf and R. Memming, Appl. Surf. Sci., 1987, 27, 423.

61 L. Spanhel, M. Hasse, H. Weller and A. Henglein, J. Am. Chem. Soc., 1987, 109, 5649.

62 X. Wang, J. Liu, H. Du and J. D. Miller, Langmuir, 2010, 26, 3407.

63 M. Taguchi, S. Takami, T. Naka and T. Adschiri, Cryst. Growth Des., 2009, 9, 5297.

64 M. S. Bakshi, P. Thakur, P. Khullar, G. Kaur and T. S. Banipal, Cryst. Growth Des., 2010, 10, 1813.

65 B. Nikoobakht and M. A. El-Sayed, Langmuir, 2001, 17, 6368.
66 X. W. Du, Y. S. Fu, J. Sun, X. Han and J. Liu, Semicond. Sci. Technol., 2006, 21, 1202.

67 J. Degenhardt and A. J. McQuillan, Langmuir, 1999, 15, 4595.

68 V. Delgado, F. Gonzalez-Caballero, R. J. Hunter, L. K. Koopal and J. Lyklema, Pure Appl. Chem., 2005, 77, 1753.

69 R. Atkin, V. S. J. Craig, E. J. Wanless and S. Biggs, J. Colloid Interface Sci., 2003, 266, 236.

70 J. Du, B. Jiang, J. Xie and X. Zeng, J. Dispersion Sci. Technol., 2001, 22, 529.

71 D. P. S. Negi and T. I. Chanu, Nanotechnology, 2008, 19, 465503.

72 K. Pita and Q. V. Vu, Energy Transfer from Silicon NCs to $\mathrm{Er}^{3+}$ Ions Embedded in Silicon Oxide Matrix, Nanocrystal, ed. Yoshitake Masuda, 2011, Ch. 17, pp. 437-458, InTech, Croatia.

73 A. Bol and A. Meijerink, J. Phys. Chem. B, 2001, 105, 10203.

74 Y. Wang and N. Herron, J. Phys. Chem., 1991, 95, 525.

75 N. Chestnoy, T. D. Harris, R. Hull and L. E. Brus, J. Phys. Chem., 1986, 90, 3393.

76 W. Gopel and U. Lampe, Phys. Rev. B, 1980, 22, 6441.

77 W. Gopel, G. Rocker and R. Feierabend, Phys. Rev. B, 1983, 28, 3427.

78 M. Kuno, J. K. Lee, B. O. Dabbousi, F. V. Mikulec and M. G. Bawendi, J. Chem. Phys., 1997, 106, 9869.

79 K. D. Kepler, G. C. Lisensky, M. Patel, L. A. Sigworth and A. B. Ellis, J. Phys. Chem., 1995, 99, 16011.

80 X. L. Diao, Y. S. Xia, T. L. Zhang, Y. Li and C. Q. Zhu, Anal. Bioanal. Chem., 2007, 388, 1191.

81 A. Kumar and S. Kumar, J. Photochem. Photobiol., A, 1992, 69, 91.

82 D. E. Fogg, L. H. Radzilowski, R. Blanski, R. R. Schrock and E. L. Thomas, Macromolecules, 1997, 30, 417.

83 J. G. C. Veinot, J. Galloro, L. Pugliese, V. Bell, R. Pestrin and W. J. Pietro, Can. J. Chem., 1998, 76, 1530. 